A N N A L E S Annales de Bretagne et des Pays de l'Ouest

Anjou. Maine. Poitou-Charente. Touraine

111-3 | 2004

Alcuin de York à Tours

\title{
Saint-Martin de Tours et les chancelleries carolingiennes
}

Mark Mersiowsky

\section{CpenEdition}

Journals

Édition électronique

URL : http://journals.openedition.org/abpo/1218

DOI : $10.4000 / a b p o .1218$

ISBN : 978-2-7535-1495-9

ISSN : 2108-6443

Éditeur

Presses universitaires de Rennes

Édition imprimée

Date de publication : 20 septembre 2004

Pagination : 73-90

ISBN : 978-2-7535-0053-2

ISSN : 0399-0826

\section{Référence électronique}

Mark Mersiowsky, « Saint-Martin de Tours et les chancelleries carolingiennes », Annales de Bretagne et des Pays de l'Ouest [En ligne], 111-3 | 2004, mis en ligne le 20 septembre 2006, consulté le 02 mai 2019. URL : http://journals.openedition.org/abpo/1218; DOI : 10.4000/abpo.1218 


\title{
Saint-Martin de Tours et les chancelleries carolingiennes
}

\author{
Mark MERSIOWSKY \\ Chargé de recherche aux Monumenta Germaniae Historica (Munich) \\ et Privatdozent à l'Université de Stuttgart
}

Presque mille ans durant, les murs de Saint-Martin de Tours renfermèrent une série exceptionnelle de diplômes impériaux de l'époque carolingienne $^{1}$, dont quelques-uns scellés d'une bulle d'or ${ }^{2}$. Le trésor de chartes de la collégiale resta presque intact jusqu'à la Révolution. D'après un procès-verbal :

« Le 27 brumaire (17 novembre), l'an II de la République Française... les titres des priviléges et des droits ci-devant seigneuriaux ont été mis en deux tombereaux et conduits, en présence du Conseil du district de Tours, sur la place Nationale... Les titres ont été jetés dans le dit bûcher et le feu y a été mis par le représentant du peuple, les présidents des autorités constituées et les chefs des corps militaires, en présence du public qui a témoigné la plus grande satisfaction de cet acte de justice, par les cris répétés de "Vive la République!" et par des danses réitérées autour du feu de joie public qui a été allumé à l'heure de quatre après-midi et a duré jusqu'à dix, à la garde d'un piquet de vingt-cinq grenadiers, qui... se sont donné tous les soins pour qu'il ne restât aucun vestige de ces titres injurieux aux droits de l'homme et ont entretenu le feu avec une activité nécessaire, vu la pluie continuelle, en présence et sous la surveillance des citoyens Rougeot, archiviste du district, et Gollineau, son collègue... qui ont dressé le dit procès verbal ${ }^{3}$."

1. A. GIRY, Notices..., p. 73-81 ; G. TESSIER, "Les diplômes... ", p. 683. Je tiens à remercier Ph. Depreux pour son aide dans la révision du texte français.

2. Paris, Bibliothèque nationale de France, Coll. Baluze $76, \mathrm{f}^{\circ} 28 \mathrm{r}^{\circ}$; cf. L. DE GRANDMAISON, "Les bulles... »; Die Regesten des Kaiserreichs (BM2), p. 263-264 (voir BM2 629); G. TESSIER, "Les diplômes... ", p. 687; G. Tessier, Diplomatique..., p. 81. Voir aussi H. BRESSLAU, « Zur Lehre... "; A. EITEL, Ueber Blei- und Goldbullen..., p. 40-45; M. MERSIOWSKY, "Graphische Symbole... ", p. 374-375.

3. É. MABILLE, La pancarte..., p. 24-25 n. 1. Je n'ai pas pu utiliser P. Gasnault, Étude sur les chartes de Saint-Martin de Tours des origines au milieu du XII siècle, thèse de l'École des chartes, 1953, mais Hélène Noizet m'a donné le chapitre 3 de sa thèse de doctorat; je l'en remercie cordialement. H. NOIZET, Pratiques spatiales, représentations de la ville et fabrique urbaine de Tours du IX $X^{e}$ au XIII siècle : chanoines, moines et lä̈cs à Saint-Martin et à Saint-Julien, 3 vol., thèse de doctorat soutenue à l'université de Tours le 9 décembre 2003, sous la direction d'Henri Galinié, 1, p. 78-81. 
Le feu de joie public de l'an II arrachera des larmes à tout jamais à tous les diplomatistes qui s'occupent de l'époque carolingienne. D'après l'inventaire sommaire qui vient à la suite dudit procès-verbal, on détruisit entre autres

" une liasse de chartes en latin qui sont des priviléges accordés par Charlemagne. Autre, des priviléges accordés par Charles le Chauve. Autre, des priviléges accordés par les rois Louis, Charles et Philippe, des IX, X, XI et $\mathrm{XII}^{\mathrm{e}}$ siècles $^{4}$ ".

En plus on a brûlé

" un Registre en parchemin intitulé : Pean Gastineau, contenant les statuts, priviléges et droits du ci-devant chapitre de St-Martin, un autre registre intitulé Pancarte Noire, contenant les priviléges et droits du dit ci-devant chapitre $^{5}$ ".

À cause de ce feu de joie de novembre 1793, il ne sera jamais possible de se livrer à une analyse approfondie sur Saint-Martin de Tours et les chancelleries carolingiennes. Depuis les travaux de Michael Tangl et Hans-Walter Klewitz, les diplomatistes s'accordent à ne pas considérer la chancellerie comme une institution bureaucratique ${ }^{6}$. Sur la chancellerie " nous ne savons guère plus que ce que les diplômes eux-mêmes nous apprennent ${ }^{7}$ ", notre information étant plus ou moins limitée aux noms de personnages qui sont mentionnés dans les notes tironiennes et qui apposèrent leur nom dans la formule de recognition au bas des actes; " il est par conséquent préférable de n'y reconnaître que l'ensemble des notaires ${ }^{8}$ ". Si nous avons les originaux, nous pouvons faire des comparaisons d'écritures et d'éléments figurés, nous pouvons voir si la personne qui écrivit le diplôme travaillait fréquemment dans ou pour la chancellerie, ou si c'était une main qui écrivait des actes privés pour sa collégiale; nous pouvons aussi déchiffrer les notes tironiennes ${ }^{9}$. Si les documents ne sont pas transmis en original, les possibilités sont plus faibles. Pour les actes transmis en copie, on ne peut analyser que les caractères rédactionnels des actes. De plus, il faut toujours s'attendre à des retouches et à des faux ${ }^{10}$.

Si l'on veut quand même parler de Saint-Martin de Tours et des chancelleries carolingiennes, il faut partir des travaux de Georges Tessier.

4. É. MABILLE, La pancarte..., p. 25 n. 1.

5. Ibidem.

6. L. PERRICHET, La grande chancellerie ... ; J. FleCKENSTEIN, Die Hofkapelle ..., p. 74-86; cf. R.-H. BautieR, " La chancellerie... ", p. 8-9; O. Guyotjeannin, J. Pycke et B.-M. Tock, Diplomatique..., p. 223-225; Ph. Depreux, Prosopographie de l'entourage de Louis le Pieux..., p. 13-16.

7. R.-H. Bautier, " La chancellerie... ", p. 8, déjà dans $\dagger$ F. Grat, J. DE Font-ReaulX, $\dagger$ G. TeSSIER et R.-H. BAUTIER (éd.), Recueil des actes de Louis II..., p. LVII.

8. Ph. Depreux, Prosopographie de l'entourage de Louis le Pieux..., p. 16.

9. Cf. T. Sickel, Lehre von den Urkunden..., p. 368-374; O. GUYOTJEAnnin, J. Pycke et B.-M. Тоск, Diplomatique..., p. 69-70.

10. Cf. T. SickEL, Lehre von den Urkunden..., p. 374-388; O. GuYOTJEANnin, J. PyCKE et B.-M. Тоск, Diplomatique..., p. 271-328. 
Georges Ernest Prosper Marie Tessier, né en 1891, décédé le 7 décembre 1967, plus de trente ans professeur de diplomatique à l'École des Chartes, membre de l'Académie des inscriptions et belles-lettres à partir de 1953 et, à compter de 1964, secrétaire perpétuel, a publié comme œuvres principales l'édition des diplômes de Charles le Chauve et son livre sur la diplomatique royale française ${ }^{11}$. Son intérêt n'était pas du tout le fruit du hasard. Parmi les diplômes de Charles le Chauve, ceux de Saint-Denis et de SaintMartin de Tours posaient des problèmes très importants, auxquels Tessier se consacra d'une manière exemplaire. En 1946, son article sur les originaux et pseudo-originaux carolingiens du chartrier de Saint-Denis porta sur un grand nombre d'originaux et de pseudo-originaux conservés; en 1951, Tessier s'occupa des actes de Saint-Martin ${ }^{12}$.

Malgré les travaux de Tessier, beaucoup de problèmes demeurent en ce qui concerne les plus anciens actes de Saint-Martin. Pour les diplômes de Pépin le Bref, Carloman et Charlemagne, l'édition de E. Mühlbacher et Michael Tangl n'est pas seulement vieillie; elle ne contient aucune histoire de la chancellerie et les observations paléographiques ne sont pas commentées ${ }^{13}$. L'édition des actes de Louis le Pieux manque encore et l'état de la recherche n'est pas satisfaisant ${ }^{14}$. Mis à part cela, le problème fondamental, c'est la transmission des actes de Saint-Martin. Les originaux sont détruits. La plupart des savants du XVII ${ }^{\mathrm{e}}$ et XVIII ${ }^{\mathrm{e}}$ siècle ne pouvait pas - ou ne voulait pas - les collationner; ces savants travaillaient avec les cartulaires médiévaux dont le plus important était la Pancarte noire ${ }^{15}$. Ce manuscrit, aussi appelé la " Grande Pancarte " (Pancarta magna), était un in-folio d'assez grande dimension. Il comprenait 157 feuillets de parchemin, l'écriture était disposée sur deux colonnes; souvent on trouvait des notes tironiennes. La Pancarte noire était reliée avec deux ais de bois recouverts d'un cuir noir. À l'époque, elle était enchaînée, grâce à une chaîne de fer, dans le trésor de la collégiale. Le cartulaire fut rédigé vers l'année 1137 et renfermait cent cinquante documents. Il fut détruit le 17 novembre $1793^{16}$. En ce qui concerne les plus anciens actes, les autres cartulaires médiévaux, de même que les copies des érudits du XVII ${ }^{\mathrm{e}}$ et XVIII ${ }^{\mathrm{e}}$ siècle, dépendaient de la Pancarte noire ${ }^{17}$. Émile Mabille a pu reconstruire ce précieux cartulaire en 1866.

11. G. Tessier (éd.), Recueil des actes de Charles II..., 1-3; G. TesSIER, Diplomatique ... ; cf. aussi "Bibliographie des travaux de Georges Tessier ", p. 73.

12. G. TESSIER, "Originaux... »; G. TESSIER, "Les diplômes... ».

13. E. Muhlbacher (éd.), Die Urkunden der Karolinger, 1 (MGH DD Kar. 1). Pour la critique, cf. déjà W. ERBEN, « Zur Herausgabe... ", p. 535, 544-545.

14. Ph. DepreuX, « Die Kanzlei... »; M. MersiowsKY, «Zur Edition... ». Depuis l'année dernière, c'est Theo Kölzer qui est responsable pour l'édition et je le soutiens dans le cadre de mes fonctions aux MGH.

15. É. Mabille, La pancarte..., p. 37-51; A. GiRY, Notices ..., p. 75-80; G. Tessier, « Les diplômes... ", p. 683; H. NoIzet, Pratiques... (cf. supra n. 3), 1, p. 78-81.

16. É. MABILLE, La pancarte..., p. 10-21; A. GirY, Notices..., p. 74; le chiffre de 150 document d'après Gasnault chez H. NoIzeT, Pratiques...(cf. supra n. 3), 1, p. 81-82.

17. É. MABILLE, La pancarte ..., p. 3-10, 21-24; MGH DD Kar. 1, p. 116; G. TESSIER, "Les diplômes... ", p. 683-684; H. NoIzET, Pratiques...(cf. supra n. 3), 1. 
À la faveur de la constitution d'un cartulaire, les textes des diplômes sont souvent remaniés. Il faut toujours se demander : "Quelles informations sont transférées de la source à la cible, de l'original à la copie ${ }^{18}$ ? " Certainement, il y eut au moins des retouches, mais aussi des falsifications dans le chartrier de Saint-Martin ${ }^{19}$. D'après les copies du XvII siècle, nous savons que la Pancarte noire était un cartulaire figuré d'une qualité extraordinaire $^{20}$ (figure 1). Normalement, les cartulaires du Moyen Âge ne contiennent que les monogrammes, les signes - peut-être complétés par la main du souverain - portant son nom : un signe du pouvoir ${ }^{21}$. En revanche, la pancarte noire présentait aussi les chrismes et les ruches, les signes des notaires et scribes. Les dessins les plus minutieux tirés de la Pancarte noire se trouvent dans un manuscrit en papier de la collection Ashburnham, aujourd'hui à Florence. Le copiste du XvII siècle qui a exécuté cette copie très soignée reste inconnu ${ }^{22}$. Très longtemps, les cartulaires ne furent considérés qu'en tant que transmetteurs de textes ${ }^{23}$. Le cartulariste du XII e siècle à Tours n'a pas négligé de donner les cotes qui se trouvaient à l'époque au dos de l'original ${ }^{24}$ : huius rescripti privilegium invenies cum tali signo ${ }^{25}, \ldots$ de hoc rescripto habemus privilegium cum tali signo ${ }^{26}$ ou hoc est rescriptum Ludovici imperatoris cum tali signo ${ }^{27}$; chaque fois, le dessin du signe suit. Malheureusement, tous les actes ne sont pas surmontés de leur cote; mais on peut supposer que le classement du chartrier de Saint-Martin de Tours fut associé à la confection de la pancarte noire ${ }^{28}$. D'après les dessins, on peut étudier la pratique médiévale de cotation à Tours. Comme souvent au Moyen Âge, l'archiviste utilisait un vieux

18. L. MORELLE, "De l'original à la copie... ", p. 91.

19. MGH DD Kar. 1, p. 116 n 81 n. i; G. TESSIER, « Les diplômes... ", p. 683-684; G. TESSIER (éd.), Recueil des actes de Charles II..., 1, p. 174, 178, 182, 301, G. Tessier (éd.), Recueil des actes de Charles II..., 2, p. 34-35, 48, 201; H. Noizet, Pratiques... (cf. supra n. 3), 1, p. 85-91.

20. A. GiRY, Notices..., p. 74; É. MABILLE, La pancarte..., p. 15. Certains signes graphiques sont copiés dans les manuscrits suivants : Paris, Bibliothèque nationale de France, ms. lat. 12683, Coll. Dupuy 657; les manuscrits les plus importants en ce qui concerne les signes graphiques sont Florence, Biblioteca Laurenziana, Ms. Ashburnham 1836, et Paris, Bibliothèque nationale de France, ms. lat. 17709. J'ai utilisé des photos colligées pour l'édition des diplômes de Louis le Pieux.

21. L. MORELLE, “ De l'original à la copie... », p. 95-97. Pour le monogramme comme signe de pouvoir, cf. P. E. Schramm, Herrschaftszeichen..., p. 18; M. MerSIowsKY, "Graphische Symbole... ", p. 350-353, 367, 381 ; O. GUYOTJEANNIN, «Le monogramme... », p. 293, 305.

22. Florence, Biblioteca Laurenziana, Ms. Ashburnham 1836; cf. L. DelisLE, « Notice des manuscrits... ", p. 89-93; cf. A. GIRY, Notices..., p. 75-76. Pour la vie de Guillaume-BrutusTimoléon Libri-Carrucci (1803-1869) et les vols des manuscrits dans les bibliothèques publiques de France, cf. L. DelisLE, Bibliothèque Nationales. Catalogue des manuscrits des fonds Libri..., p. V-LXXXII; G. FuMAGalLI, Guglielmo Libri...

23. P. GEARY, « Entre gestion et gesta ", p. 13-14.

24. On ce trouve souvent, cf. L. MORELLE, « De l'original à la copie... ", p. 92-93.

25. Florence, Biblioteca Laurenziana, Ms. Ashburnham 1836, $\mathrm{f}^{\circ} 32 \mathrm{v}^{\circ}$.

26. Ibid. $\mathrm{f}^{\circ} 37 \mathrm{r}^{\circ}$.

27. Ibid., $\mathrm{f}^{\circ} 56 \mathrm{r}^{\circ}$.

28. Cf. L. MORELLE, " De l'original à la copie... », p. 93. 
système de repérage (marquage avec des signes, des marques visuelles distinctives ${ }^{29}$, ici des signes graphiques abstraits et lettres) (figures 1 à 3). Les souscriptions du cartulaire reproduisent les divers notations dorsales des originaux perdus. Ces courtes analyses de l'acte étaient rédigées partiellement en notes tironiennes ${ }^{30}$. L'analyse du manuscrit Ashburnham est confirmée par les débris du chartrier de Saint-Martin. Heureusement, deux copies figurées du $\mathrm{X}^{\mathrm{e}}$ siècle d'un original perdu de Louis II le Bègue sont conservées à la Bibliothèque nationale de France ${ }^{31}$. Au dos de $\mathrm{B}$ et $\mathrm{C}$, se trouvent des mentions contemporaines écrites en partie en notes tironiennes ${ }^{32}$. B porte aussi la phrase : XI ${ }^{\circ}$ quaterno invenies rescriptum cum tali signo, d'une main du XII ${ }^{\mathrm{e}}$ siècle; on y trouve un dessin comparable aux dessins de la copie Ashburnham ${ }^{33}$. Dans ce cas, la transcription du diplôme manque dans le manuscrit Ashburnham ${ }^{34}$.

Georges Tessier a montré que parmi les diplômes de Charlemagne, Louis le Pieux et Charles le Chauve pour Saint-Martin, certains actes étaient rédigés d'après un modèle de chancellerie, mais que le texte de la plupart relève d'un style plus libre ${ }^{35}$. Pour Saint-Denis, il avait eu la possibilité d'analyser les originaux, et il identifia dans son étude classique sur le chartrier de SaintDenis des chartes écrites par des scribes de la chancellerie et des chartes écrites par des mains sandionysiennes ${ }^{36}$. Les observations de Tessier que j'ai pu contrôler et approfondir pour les diplômes des Louis le Pieux ${ }^{37}$ montent que les moines de Saint-Denis ont écrit eux-mêmes un certain nombre de leurs diplômes; souvent, leur dictamen diverge du modèle commun. Philippe Lauer a constaté que le style des actes de Charles III le Simple pour Saint-Martin de Tours différait de celui des autre diplômes. Lauer ne se prononça pas sur la possibilité d'une interpolation par le cartulariste ou sur celle d'une rédaction des actes par les chanoines ${ }^{38}$. Pour Louis II le Bègue, on a accepté l'idée que les frères de Saint-Martin avaient établi les actes ${ }^{39}$.

29. Pour cette technique, cf. O. GUYOTJEANNIN, " La tradition de l'ombre... ", p. 83-86.

30. En ce qui concerne les notions dorsales de Saint-Martin, cf. P. GASNAULT, « Les actes privés... ", p. 34 n. 5. Pour la pratique médiévale, cf. L. Morelle, " De l'original à la copie... ", p. 92f; O. GUYOTJEANNIN, " La tradition de l'ombre... ", p. 82, 87.

31. Paris, Bibliothèque nationale de France, ms. lat. 8837, $\mathrm{f}^{\circ} 59,60$, cf. Catalogue général..., p. 22f; † F. GRAT, J. DE FONT-REAULX, † G. TESSIER et R.-H. BAUTIER (éd.), Recueil des actes de Louis II..., n 12, p. 28-33; H. Nolzet, Pratiques... (cf. supra n. 3), 1, p.88-89.

32. Paris, Bibliothèque nationale de France, ms. lat. 8837, $\mathrm{f}^{\circ} 59 \mathrm{v}^{\circ}, 60 \mathrm{v}^{\circ}, \dagger$ F. GRAT, J. DE FONT-REAULX, $\uparrow$ G. TeSSER et R.-H. BAUTIER (éd.), Recueil des actes de Louis II. .., n. 12, p. 29 n. 1-2.

33. Paris, Bibliothèque nationale de France, ms. lat. $8837, \mathrm{f}^{\circ} 59 \mathrm{v}^{\circ}, \uparrow \mathrm{F}$. GRAT, J. DE FonTReaulX, $\dagger$ G. Tessier et R.-H. Bautier (éd.), Recueil des actes de Louis II..., n 12, p. 29 n. 1.

34. $\dagger$ F. Grat, J. DE Font-ReaulX, $\dagger$ G. Tessier et R.-H. Bautier (éd.), Recueil des actes de Louis II..., n ${ }^{\circ} 12$, p. 29 n. 3.

35. G. Tessier (éd.), Recueil des actes de Charles II..., 3, p. 110-111; p. GASNAUlt, " Les actes privés... ", p. 29; H. Noizet, Pratiques... (cf. supra n. 3), 1, p. 91-99.

36. G. Tessier, "Originaux..." ".

37. M. MERSIOWSKY, "Graphische Symbole...», p. 339-340.

38. P. LAUER (éd.), Recueil des actes de Charles III..., p. LCVI.

39. $\dagger$ F. Grat, J. De Font-ReaulX, † G. Tessier et R.-H. Bautier (éd.), Recueil des actes de Louis II..., p. LXIX, LXXXIX. 
En regardant la confirmation d'immunité accordée par Louis le Pieux à Saint-Martin en 816, il est hors de doute que l'acte fut dicté par le destinataire. Elle est plus raffinée que les autres privilèges, la langue est ornée, et comme base on a utilisé les diplômes de Charlemagne pour Saint-Martin - un cas isolé, parce que normalement la chancellerie évitait de reprendre les formulaires vieillis ${ }^{40}$. Ainsi, dans le vocabulaire, on observe certaines particularités. L'expression patronis nostri se trouve dans 4 diplômes de Louis le Pieux pour Saint-Denis ${ }^{41}$ et dans 7 diplômes pour Saint-Martin ${ }^{42}$, outre deux diplômes pour Cormery, qui à l'époque faisait partie de Saint-Martin ${ }^{43}$. Deux diplômes pour Saint-Denis ${ }^{44}$ et un pour Cormery sont conservés en original. Pendant le règne de Louis le Pieux en Aquitaine, l'expression fut une fois utilisée pour Saint-Hilaire à Poitiers ${ }^{45}$; de 814 à 840, on ne la trouve que dans un diplôme controversé pour Saint-Remi, peut-être aussi rédigé par le bénéficiaire ${ }^{46}$, et dans un autre pour Saint-Philibert - ce diplôme est conservé en original ${ }^{47}$.

Bien que les originaux de Saint-Martin de Tours soient perdus, les dessins de la Pancarte noire permettent d'identifier les signes graphiques typiques des notaires qui ont reconnu les diplômes. La confirmation d'immunité accordée par Louis le Pieux le 30 août 816, dont l'original est perdu, portait la recognition de Helisachar (Helisachar recognovi), avec le chrisme dans sa forme normale et une ruche variée du deuxième type de Helisachar ${ }^{48}$ (figures 4 et 5). Le chrisme au début du texte ne correspond pas aux chrismes des personnes faisant habituellement la recognition à la chancellerie de Louis le Pieux ${ }^{49}$; il est dû au scribe anonyme. Dans le diplôme du 30 août 816, on peut facilement reconnaître la ruche de Durand ${ }^{50}$. Le dessin rend même possible de reconnaître les notes tironiennes de l'original perdu ${ }^{51}:$ Durandus diaconus advicem Helisachar recognovi et subscripsi ${ }^{52}$ (figure 6). La copie du

40. G. TESSIER, "Les diplômes... ", p. 684-685.

41. $\mathrm{BM}^{2} 551,552,554,906$.

42. $\mathrm{BM}^{2} 629$ et K. ZEUMER (éd.), Formulae merowingici..., n ${ }^{\circ} 29, \mathrm{BM}^{2}$ 631, 632, 649, 896, 909,910 .

43. $\mathrm{BM}^{2} 886,967$.

44. $\mathrm{BM}^{2}$ 551, 552 (Saint-Denis) = F. Lot et P. LAuER (éd.), Diplomata Karolinorum, $2^{1}$, pl. 3 et 4, BM 967 (Cormery) = F. Lot et P. LAUER (éd.), Diplomata Karolinorum, 22, pl. 49.

45. $\mathrm{BM}^{2} 516$.

46. $\mathrm{BM}^{2}$ 801, cf. Ph. DePreuX, «Zur Echtheit... ».

47. $\mathrm{BM}^{2} 875$ = F. Lot et P. LAUER (éd.), Diplomata Karolinorum, 22, pl. 43.

48. $\mathrm{BM}^{2}$ 629, Florence, Biblioteca Laurenziana, Ms. Ashburnham 1836, $\mathrm{f}^{\circ} 6 \mathrm{r}^{\circ}-\mathrm{v}^{\circ}$, pour les chrismes et ruches de Helisachar, cf. M. MERSIOWSKY, "Graphische Symbole... ", p. 347, 371-372, avec pl. 18 p. 360 et pl. 48 p. 365.

49. Florence, Biblioteca Laurenziana, Ms. Ashburnham 1836, $\mathrm{f}^{\circ} 4 \mathrm{v}^{\circ}$, cf. M. MERSIOWSKY, "Graphische Symbole...", pl. 5-25, p. 358-361.

50. $\mathrm{BM}^{2}$ 632, Florence, Biblioteca Laurenziana, Ms. Ashburnham 1836, $\mathrm{f}^{\circ} 38 \mathrm{r}^{\circ}$, pour les ruches de Durand, cf. M. Mersiowsky, " Graphische Symbole... ", p. 370-371, avec pl. 33 p. 362 et pl. 40 p. 364 .

51. Pour la critique des dessins des notes tironiennes dans les cartulaires M. TANGL, "Forschungen... ", p. 361-363.

52. Les notes utilisés par Durandus sont expliqués chez M. TANGL, "Die tironischen Noten... ", p. 304, cf. les planches chez M. MERSIOWSKY, "Zur Edition... ", p. 340. 
diplôme du 14 novembre 832 montre bien le chrisme et la ruche de Hirminmaris $^{53}$ (figure 7). Les observations confirment qu'une partie des diplômes de Louis le Pieux pour Saint-Martin avait été rédigée par les chanoines, mais reconnue par la chancellerie. Si le chrisme était vraiment au début du diplôme du 14 novembre 832 comme dans la copie de Florence ${ }^{54}$ (figure 8), cela veut dire que Hirminmaris écrivit lui même le diplôme!

Mais il y avait aussi des problèmes : par exemple, la ruche de Rado dans la confirmation des biens de 775 , accordée par Charlemagne ${ }^{55}$. Rado a reconnu 19 actes de Charlemagne, dont 9 conservés - d'après les travaux de Mühlbacher - en original, pseudo-original ou copie figurée très ancienne ${ }^{56}$. Si l'on regarde les recognitions de Rado dans les originaux, il y a un groupe certainement autographe ${ }^{57}$. Dans un diplôme, il ne subsiste que les longues hastes recourbées, la courbe supérieure de la ruche et quelques traces des lettres, mais on ne peut manquer d'y reconnaître la main de $\operatorname{Rado}^{58}$. En ce qui concerne le diplôme de 774/775 pour Saint-Denis, E. Mühlbacher a remarqué que la souscription de Rado n'était pas autographe; cependant, il a considéré cet acte comme original. Michael Tangl, déjà, dut le contredire. En fait, dans les originaux, Rado ne séparait pas les mots de la souscription et la ruche, qui est une copie mal comprise; l'emplacement du sceau ne correspond pas aux manières de la chancellerie à cette époque. L'acte doit être considéré comme une copie figurée contemporaine ${ }^{59}$. Le dessin de la ruche de Rado dans la confirmation des biens de 775 pour Saint-Martin de Tours, dans le manuscrit de Florence, ne correspond pas assez aux ruches originales de Rado; le dessin est trop simple et

53. $\mathrm{BM}^{2}$ 909, Florence, Biblioteca Laurenziana, Ms. Ashburnham 1836, $\mathrm{f}^{\circ} 38 \mathrm{v}^{\circ}, 39 \mathrm{v}^{\circ}$, pour les chrismes et ruches de Hirminmaris, cf. M. MERSIOWSKY, "Graphische Symbole...", p. 347,372 , avec pl. 21 p. 360 , pl. 34 p. 363 et pl. 49 p. 365 .

54. Florence, Biblioteca Laurenziana, Ms. Ashburnham $1836, \mathrm{f}^{\circ} 38 \mathrm{v}^{\circ}$.

55. MGH DD Kar. 1, $\mathrm{n}^{\circ}$ 97. Le dossier préparatoire pour l'édition est conservé aux archives des MGH, Munich, A 98/2 avec un dessin de la ruche de Alphons Dopsch, mais aucun notice se trouve en ce qui concerne la ruche.

56. MGH DD Kar. $1, \mathrm{n}^{\circ}$ 67, 71(copie figurée), 82, 83 (original), 84A (original), 85, 88 (original), 96, 97, 103 (original), 106, 107 (original), 113, 114, 118 (original), 121 (original), 130 (original), 141, 142, † 228, † 276 (d'après les analyses de Mühlbacher).

57. F. Lot et Ph. LAuER (éd.), Diplomata Karolinorum, 1, pl. $15=$ H. ATSMa et J. Vezin (éd.), Chartae latinae antiquiores..., 15, n 613 (MGH DD Kar. 1, n 84 A), H. von SYBEL et T. von Sickel (éd), Kaiserurkunden..., 3, pl. 2 = A. BruCKNER et R. MARICHAL (éd.), Chartae latinae antiquiores, 12, n 535 (MGH DD Kar. 1, n 103), Lot, LAUER, pl. 24 = H. ATSMA et J. VEZIN (éd.), Chartae latinae antiquiores, 16, n 620 (MGH DD Kar. 1, n 107), LOT et LAUER, pl. 25 $=$ H. ATSMa, R. Marichal et J. Vezin (éd.), Chartae latinae antiquiores, 19, n 679 (MGH DD Kar. $1, \mathrm{n}^{\circ} 118$ ), H. von SyBEL et T. von Sickel (éd), Kaiserurkunden ..., 3, pl. 3 = A. BRUCKNER et R. Marichal (éd.), Chartae latinae antiquiores, 12, n 537 (MGH DD Kar. 1, n 121), A. BRUCKNER et R. MARICHAL (éd.), Chartae latinae antiquiores, 2, $\mathrm{n}^{\circ} 157=$ Albert BRUCKNER (éd.), Diplomata Karolinorum, 1, n 4 (MGH DD Kar. 1, n ${ }^{\circ} 130$ ). Sur les souscriptions de l'époque carolingienne, cf. P. WoRM, Karolingische Rekognitionszeichen...

58. F. Lot et Ph. Lauer (éd.), Diplomata Karolinorum, 1, pl. $14=$ H. AtSMA et J. Vezin (éd.), Chartae latinae antiquiores, $16, \mathrm{n}^{\circ} 627$ (MGH DD Kar. 1, $\mathrm{n}^{\circ} 83$ ).

59. Lot et Lauer, pl. 17 = H. Atsma et J. Vezin (éd.), Chartae latinae antiquiores, $16, \mathrm{n}^{\circ} 621$ (MGH DD Kar. 1, $\mathrm{n}^{\circ} 88$ ), cf. la contradiction de Tangl, p. 563-564. 
il manque le mot et entre la souscription et la ruche ${ }^{60}$. Parce que les autres dessins étaient plus raffinés, il faut soupçonner que la copie dans la Pancarte noire a été faite d'après un pseudo-original interpolé ${ }^{61}$ (figure 1). Dans un autre cas, c'est évident. Le signum de Louis le Pieux en lieu et place du monogramme dans le texte de la confirmation d'immunité du $1^{\text {er }}$ juillet 817 rappelle une combinaison entre les signa des actes privés et le Chi-Rho et n'apparaît dans aucun original de Louis le Pieux ${ }^{62}$ (figure 9).

Pendant le règne de Charlemagne et celui de Louis le Pieux, la rédaction des actes par le destinataire était un cas exceptionnel; normalement, il existait des relations très étroites entre le destinataire et le palais - sinon la chancellerie.

Hitherius, déjà actif dans la chancellerie de Pépin le Bref, dirigeant de celle de Charlemagne jusqu'en 776, était abbé de Saint-Martin de Tours depuis $775^{63}$. Fridugise, un élève d'Alcuin, avait des liens avec la cour de Charlemagne. Depuis 808, il est attesté comme abbé de Saint-Martin de Tours; en 819, il fut nommé archichancelier et demeura à la tête de la chancellerie jusqu'en 832. Bien qu'il n'ait pas fait la récognition des diplômes, les mentions en notes tironiennes confirment bien qu'il jouait un rôle actif dans la chancellerie ${ }^{64}$. Théoton, son successeur comme archichancelier en 833, était certainement abbé de Saint-Martin de Marmoutier, peut-être aussi de Saint-Martin de Tours ${ }^{65}$. Il est probable que les liens avec la chancellerie se relâchèrent après 834 , sous Hugues; il était - comme Fridugise abbé de Saint-Bertin, mais pas de Saint-Martin ${ }^{66}$.

60. Florence, Biblioteca Laurenziana, Ms. Ashburnham 1836, $\mathrm{f}^{\circ} 37 \mathrm{r}^{\circ}=$ MGH DD Kar. 1, n 97 p. $139-140$.

61. Le texte est interpolé, cf. H. Nolzet, Pratiques... (cf. supra n. 3), 1, p. 99-100. On peut imaginer que le pseudo-original a réduit les signes graphiques complexes comme les copies figurées de Saint-Martin, cf. Paris, Bibliothèque nationale de France, ms. lat. 8837, $\mathrm{f}^{\circ}$ 59, $60=\uparrow$ F. Grat, J. DE Font-ReaulX, $\dagger$ G. Tessier et R.-H. Bautier (éd.), Recueil des actes de Louis II..., n. 12, p. 28-33 = F. LoT et P. LAUER (éd.), Diplomata Karolinorum, 8, pl. 30*$30^{* *}$. Avant ses propres travaux sur le chartrier de Saint-Martin de Tours Georges Tessier a soupçonné les modèles de la Pancarte noire comme pseudo-originaux, cf. G. TESSIER (éd.), Recueil des actes de Charles II..., 1, n 61 p. 174, n 62 p. 178, n 63 p. $182, \mathrm{n}^{\circ} 167$ p. 440, mais il l'a corrigé, cf. G. TESSIER, "Les diplômes... ", p. 690-691; G. TESSIER (éd.), Recueil des actes de Charles II..., 2, p. 669.

62. $\mathrm{BM}^{2}$ 649, Florence, Biblioteca Laurenziana, Ms. Ashburnham 1836, $\mathrm{f}^{\circ} 34 \mathrm{r}^{\circ}$.

63. G. Tessier, «Les diplômes... », p. 684; J. Fleckenstein, Die Hofkapelle ..., p. 76-77;

G. Tessier, Diplomatique..., p. $42-43$.

64. T. SiCKEL, Lehre von den Urkunden..., p. 89-91; J. FLECKENSTEIN, Die Hofkapelle ..., p. 8183; G. Tessier, Diplomatique..., p. 44; P. Gasnault, "Les actes privés... ", p. 26-27; O. G. OEXLE, Forschungen ..., p. 39-40; R.-H. BAUTIER, « La chancellerie... », p. 13; W. VogleR, "St. Martin in Tours und St. Gallen... ", p. 121-122, 125-126; Ph. DepreuX, Prosopographie de l'entourage de Louis le Pieux..., p. 201-202.

65. T. SicKel, Lehre von den Urkunden..., p. 95-96; J. FlECKENSTEIN, Die Hofkapelle..., p. 83; G. Tessier, Diplomatique ..., p. 44; O. G. Oexle, Forschungen..., p. 40, 48; Ph. Depreux, Prosopographie de l'entourage de Louis le Pieux..., p. 387-388.

66. T. SICKEL, Lehre von den Urkunden..., p. 96-99; J. FLECKENSTEIN, Die Hofkapelle ..., p. 8384; G. Tessier, Diplomatique ..., p. 44; Ph. DePreuX, Prosopographie de l'entourage de Louis le Pieux..., p. 264-268. 
Les indications concernant les notaires sont plus ténues. Dans la liste des chanoines de Saint-Martin (datant peut-être d'entre 818 et 820 ) notée dans le premier Liber memorialis de Saint-Gall vers 830 et copiée dans le deuxième codex ${ }^{67}$, on trouve les noms d'Adalulf, notaire en 828, Ermenmarus et Hirminmaris ${ }^{68}$. Hirminmaris est attesté à la chancellerie depuis 816; après la disparition de Durand en 832, il devint le notaire et l'auteur de recognitions le plus remarquable de la chancellerie de Louis le Pieux ${ }^{69}$. Sous sa direction, certains changements furent effectués; par exemple, on évita désormais l'usage du chrisme au début des chartes ${ }^{70}$. Nous connaissons une inscriptions peut-être autographe dans le livre de fraternité de la Reichenau : HIRMINMARIS VOCOR FRATRIBUS FIDELISSIMUS ${ }^{71}$. Depuis le voyage de Theodor Sickel en 1876, on pensait que Adalleod, notaire dans la chancellerie de Louis le Germanique, provenait de SaintMartin de Tours, parce que Sickel avait trouvé le nom de Adilleodus dans la liste de chanoines de Saint-Martin ${ }^{72}$. Mais l'identification de Adilleodus avec Odilleoz, le frère de l'abbé Heito de la Reichenau, me semble plus vraisemblable, en raison de sa position dans la liste ${ }^{73}$. On ne peut pas trouver de lien entre Saint-Martin de Tours et la chancellerie de Charles le Chauve ${ }^{74}$.

Il faut encore jeter un regard sur le monument le plus important pour les relations entre la chancellerie impériale et Saint-Martin de Tours : le manuscrit latin 2718 de la Bibliothèque nationale de France, édité pour la première fois par Dom Pierre Charpentier en $1747^{75}$. Provenant de SaintMartin de Tours, il contient des textes divers : des textes théologiques et patristiques, des capitulaires de Louis le Pieux datant des années 817 à 821 et les formulae imperiales. Il s'agit de 55 textes abrégés et rendus plus ou moins anonymes à partir de diplômes provenant de la chancellerie de Louis le Pieux et destinés à divers bénéficiaires (institutions ecclésiastiques, mais aussi marchands juifs et affranchis). Pour cette raison, c'est certainement au palais de Louis le Pieux qu'on compila ces formulae ${ }^{76}$. L'analyse paléo-

67. P. Gasnault, "Les actes privés... ", p. 26-27; O. G. OeXle, Forschungen..., p. 35-37, pour la datation p. 48-51. Le fait qu'il s'agit de deux livres a été montré par K. ScHMID, "Das ältere und das neuentdeckte jüngere... "; facsimilés p. 112-113, 209-210.

68. O. G. OEXLE, Forschungen..., p. 37 n. 165, 198, 209, K. Schmid, "Versuch... ", p. 113. Pour les relations entre Saint-Martin et Saint Gall à l'époque carolingienne, cf. W. VogLER, " St. Martin in Tours und St. Gallen... ", p. 122-132.

69. T. SiCKEL, Lehre von den Urkunden..., p. 91-92; Ph. DePREux, Prosopographie de l'entourage de Louis le Pieux..., p. 258-260.

70. M. MERSIOWSKY, "Graphische Symbole...», p. 342-343.

71. J. AutenRleth, D. Geuenich et K. Schmid (éd.), Das Verbrüderungsbuch..., pl. 5 B1 (e 159), p. 207.

72. O. G. OEXLE, Forschungen..., p. 35 n. 79; K. SchmID, "Versuch... ", p. 112. Theodor Sickel, Ueber Kaiserurkunden..., p. 4-5; Ph. DePREUX, Prosopographie de l'entourage de Louis le Pieux..., p. 82 avec les autres titres qui dépendent de Sickel.

73. O. G. OEXLE, Forschungen..., p. 45.

74. Ibid., p. 44.

75. P. CHARPENTIER, Alphabetum Tironianum...

76. Ed. K. Zeumer (éd.), Formulae merowingici..., p. 285-327, cf. p. 285 et pour les lieux p. 286-287. T. SicKEL, Lehre von den Urkunden..., p. 116-121. 
graphique faite par Bernhard Bischoff et Rosamond McKitterick a associé ce manuscrit à un scriptorium ayant produit un grand nombre de manuscrits de leges ${ }^{77}$; Martin Hellmann l'a compté parmi les témoins de l'école tironienne de Saint-Martin, qui demeura vivante jusqu'au $\mathrm{X}^{\mathrm{e}}$ siècle $^{78}$.

Les formulae sont dispersées en huit ensembles, souvent en combinaison avec des capitulaires; la plupart des textes est de nature théologique ${ }^{79}$. Entre les diplômes, on trouve aussi des formulaires d'autres chartes ${ }^{80}$ : une charte de franchise ${ }^{81}$, un échange de biens entre un abbé et un comte ${ }^{82}$, suivi d'une liste d'impôts, et un acte privé d'Eginhard comme abbé laïque ${ }^{83}$. Les textes ne sont pas uniformes : quelquefois, on les a résolument rendus anonymes et préparés comme un formulaire ${ }^{84}$; quelquefois, on a omis certaines parties du discours diplomatique; quelquefois, on a copié les textes presque entièrement ${ }^{85}$. Cela veut dire que les textes ne sont pas rédigés d'une façon systématique, ni proprement organisée. Dans un texte, nous trouvons même une mention qui était écrite en notes tironiennes dans l'original - cela signifie que le rédacteur des formulae connaissait vraiment l'original $^{86}$. On peut dater les textes d'entre la fin des années dix du neuvième siècle et la fin des années vingt : le manuscrit fut donc écrit sous Fridugise ${ }^{87}$.

Robert-Henri Bautier et Peter Johanek ont considéré les Formulae Imperiales comme "plus un florilège d'actes réellement expédiés, compilé par quelque notaire pour son usage personnel, qu'un véritable manuel de chancellerie $^{88} »$. En fait, l'analyse codicologique confirme que le manuscrit était une collection occasionnelle, et non systématique, rédigée en utilisant

77. R. MCKITTERICK, "Zur Herstellung von Kapitularien... ", p. 12, cf. aussi H. MoRdEK, Bibliotheca capitularia..., p. 422-423; H. MORDEK, " Kapitularien und Schriftlichkeit ", p. 334337. H. MoRDEK, "Fränkische Kapitularien... ", p. 39-40; D. GANZ, " Paris BN Latin 2718... ", p. 138.

78. M. HELlmann, Tironische Noten..., p. 32 n. 93, en ce qui concerne la tradition vivante des notes tironiennes à Tours p. 22-32, cf. aussi É. MABILle, La pancarte..., p. 15; P. GASNAULT, "Les actes privés... ", p. 34-35; M. CouRTOIS, " Remarques sur les chartes originales..." ", p. 60-67.

79. La composition est décrite par H. MORDEK, Bibliotheca capitularia..., p. 422-430, cf. aussi D. GANZ, "Paris BN Latin 2718... ", p. 138-142 et p. 151.

80. P. JOHANEK, " Herrscherdiplom... ", p. 185-186.

81. K. ZEumer (éd.), Formulae merowingici..., n ${ }^{\circ} 33$, p. 311-312; cf. P. JoHANEK, "Herrscherdiplom... ", p. 187.

82. K. Zeumer (éd.), Formulae merowingici..., n ${ }^{\circ} 54$, p. 326.; cf. P. JohaneK, "Herrscherdiplom... ", p. 187-188.

83. K. ZeUMer (éd.), Formulae merowingici..., n ${ }^{\circ} 35$, p. 313; cf. P. JoHANEK, "Herrscherdiplom... ", p. 187.

84. K. ZEUMER (éd.), Formulae merowingici..., n ${ }^{\circ} 1,2,3,4,5,7,11,12,13,14,15,16,17$, 18, 19, 20, 21, 23, 25, 26, 27, 28, 32, 33, 37, 41, 42, 46, 51, 54., cf. déjà T. SicKEL, Lehre von den Urkunden..., p. 117-118.

85. K. Zeumer (éd.), Formulae merowingici..., n 33 p. $311-312$., n ${ }^{\circ} 35$ p. 313 , n 43 p. $319-320$.

86. K. ZEUMER (éd.), Formulae merowingici..., n 43, p. 320 (Suizgarius ambasciavit).

87. T. SICKEL, Lehre von den Urkunden..., p. 120; K. ZEUMER (éd.), Formulae merowingici..., p. 285 ; G. TESSIER, Diplomatique..., p. 61.

88. R.-H. BAUTIER, " La chancellerie... ", p. 44; P. JOHANEK, "Herrscherdiplom... ", p. 186. 
les restes de parchemin qui se trouvaient à la chancellerie; il s'ensuit que le manuscrit est d'un format oblong. Le parchemin est inégal et écrasé, il semble avoir été conservé un certain temps sans reliure; la composition des cahiers - qui formaient déjà un ensemble avant la reliure - n'a pas d'incidence sur la mise en page du texte ${ }^{89}$. L'écriture livresque est parallèle au grand côté et semble très rapide. Les marges de gauche et de droite sont très étroites et inégales. L'espace entre les lignes est serré. Dans les formulae, l'encre et la largeur du calame changent souvent; ceci montre que les entrées furent réalisées successivement ${ }^{90}$.

Pour Sickel la chancellerie fonctionnait comme le bureau d'un ministère et Fridugise était le chef de bureau, responsable pour tous les produits de la chancellerie. Mais il n'attribua pas expressément à Fridugise les Formulae Imperiales ${ }^{91}$. Comme souvent, les recherches suivantes atténuèrent les constatations prudentes de Sickel : d'après Josef Fleckenstein, c'est Fridugise qui fit établir la collection des Formulae Imperiales ${ }^{92}$; d'après Georges Tessier, ces modèles ont été " entrepris probablement sous l'inspiration de l'archichancelier Fridugisus ${ }^{93}$... " Dans les dernières années, l'attribution à Hirminmaris a été discutée ${ }^{94}$.

Les relations du manuscrit avec Saint-Martin de Tours, dont il provient, sont évidentes. L'un des plus importants textes de Tours, la confirmation d'immunité accordée par Louis le Pieux, se trouve dans la collection - il s'agit d'un texte rédigé d'après les deux diplômes de Charlemagne pour Saint-Martin et s'écartant des formules utilisées par la chancellerie; il reste plus ou moins isolé parmi les diplômes de Louis le Pieux. Il ne sert pas souvent de modèle, mais il était très important pour Saint-Martin ${ }^{95}$. De plus, le manuscrit contient la lettre de Charlemagne à Alcuin et à la communauté de Saint-Martin ${ }^{96}$.

Dans les formulae, on trouve un acte privé d'Eginhard ${ }^{97}$. Parce que Hirminmaris a écrit et souscrit un testament sur la demande de Eginhard

89. Plus courtes que le reste du manuscrit sont Paris, Bibliothèque nationale de France, ms. lat. $2718, \mathrm{f}^{\circ} 3,4,5,11,12,13,21,22,24,64,67,84,85$; les marges inégales se trouvent $\operatorname{aux}^{\circ} 14,15,18,20,26,27,28,29,37,64,65.66,67,72,116,119,123,127,128$; cf. D. GANZ, "Paris BN Latin 2718... ", p. 138.

90. Paris, Bibliothèque nationale de France, ms. lat. $2718, \mathrm{f}^{\circ} 80 \mathrm{r}^{\circ}-80 \mathrm{v}^{\circ}, 84 \mathrm{v}^{\circ}-85 \mathrm{v}^{\circ}, 111$ $\mathrm{v}^{\circ}, 125 \mathrm{r}^{\circ}-127 \mathrm{r}^{\circ}$.

91. T. SiCKEL, Lehre von den Urkunden..., p. 75-76, 92-95,119-120; de même, cf. K. ZEUMER (éd.), Formulae merowingici..., p. 285.

92. J. Fleckenstein, Die Hofkapelle..., p. 82.

93. G. Tessier, Diplomatique..., p. 61.

94. Ph. Depreux, Prosopographie de l'entourage de Louis le Pieux..., p. 259-260;

R. MCKitTerick, "Zur Herstellung von Kapitularien... ", p. 13-14; P. JOHANEK, "Herrscherdiplom... ", p. 185; D. GANZ, "Paris BN Latin 2718... ", p. 151.

95. K. Zeumer (éd.), Formulae merowingici..., n 29, p. 307-308, cf. E. STENGEL, Diplomatik..., p. 24.

96. Paris, Bibliothèque nationale de France, ms. lat. 2718, $\mathrm{f}^{\circ} 124 \mathrm{r}^{\circ}-125 \mathrm{r}^{\circ}$; cf. H. MoRDEK, Bibliotheca capitularia..., p. 428.

97. K. ZEuMER (éd.), Formulae merowingici..., n ${ }^{\circ}$ 35, p. 313. 
en 819 (ce document est conservé dans le Codex Laureshamensis ${ }^{98}$ ), la charte pourrait nous renvoyer à Hirminmaris. La comparaison du ms. lat. 2718 avec les diplômes est difficile, parce que ce ne sont que les recognitions qui sont certainement écrites par Hirminmaris lui-même; or, il avait là recours à une écriture diplomatique. De plus, une analyse paléographique en profondeur des actes de Louis le Pieux fait toujours défaut. Hirminmaris ne semble pas avoir apprécié beaucoup les notes tironiennes; il n'y a pas eu aussi souvent recours que Durand. Toutefois, les notes tironiennes de Hirminmaris sont généralement faites très rapidement, de manière très fluide; en revanche, les notes du manuscrit sont très bien tracées, et de manière très tranquille. Je n'ai pas pu identifier la main d'Hirminmaris dans le manuscrit latin 2718, mais il faudrait approfondir les analyses de ce manuscrit fameux.

Sous Charlemagne et Louis le Pieux, les relations entre les chancelleries et Saint-Martin de Tours furent proches; même si le manuscrit ne peut pas être attribué à Hirminmaris, il reflète le rôle de cette communauté à l'époque carolingienne.

98. K GLOCKNER (éd.), Codex Laureshamensis, n. 20, p. 301-302. 
Figure 1 - Florence, Bibl. Laurenziana, Ms. Ashburnham 1836, $f^{\circ} 37 r^{\circ}$ (cliché : MGH Apparat Ludwig der Fromme, Bonn)

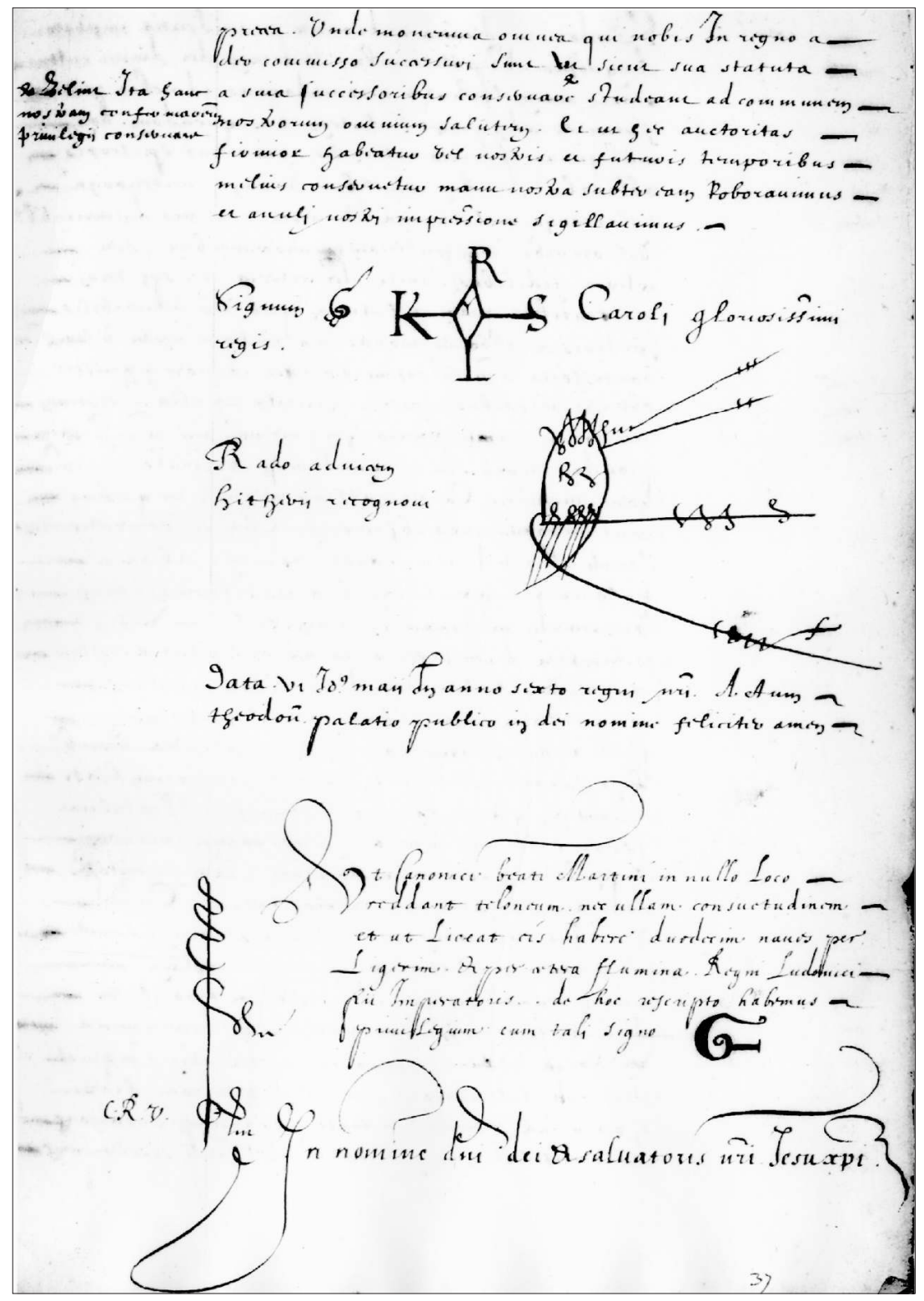


Figure 2 - Florence, Bibl. Laurenziana, Ms. Ashburnham 1836, $f^{\circ} 56 r^{\circ}$ (cliché : MGH Apparat Ludwig der Fromme, Bonn)

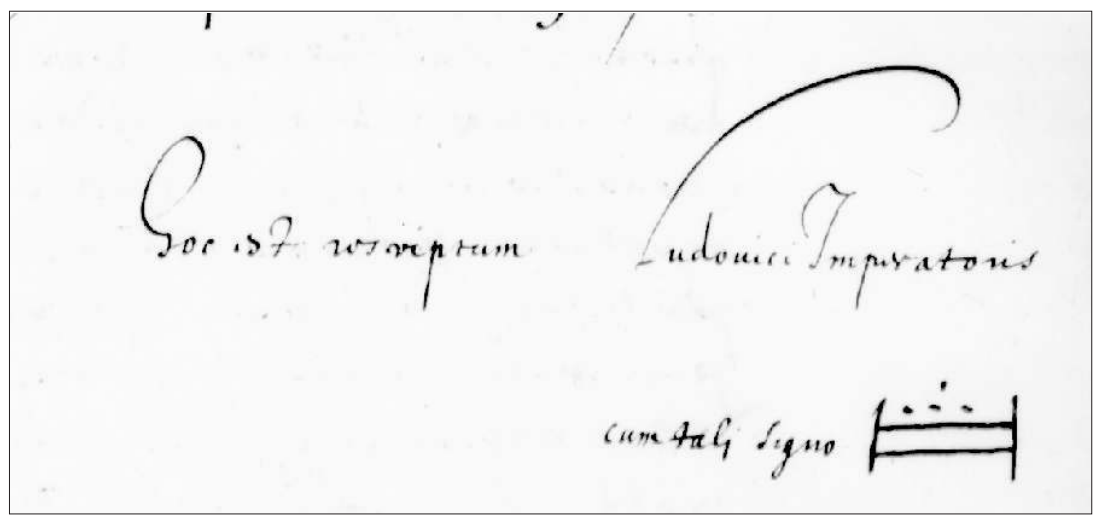

Figure 3 - Florence, Bibl. Laurenziana, Ms. Ashburnham 1836, $f^{\circ} 32 v^{\circ}$ (cliché : MGH Apparat Ludwig der Fromme, Bonn)

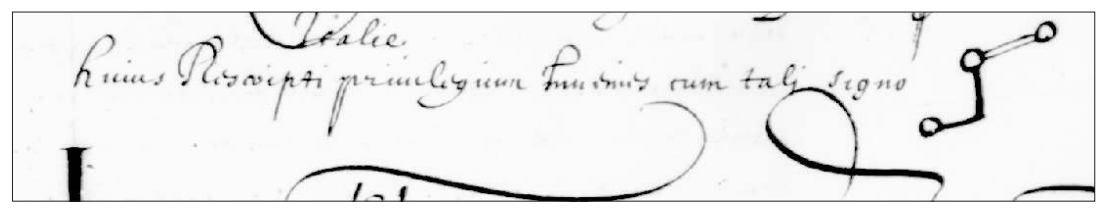

Figure 4 - Florence, Bibl. Laurenziana, Ms. Ashburnham 1836, $f^{\circ} 6 r^{\circ}$ (cliché : MGH Apparat Ludwig der Fromme, Bonn)

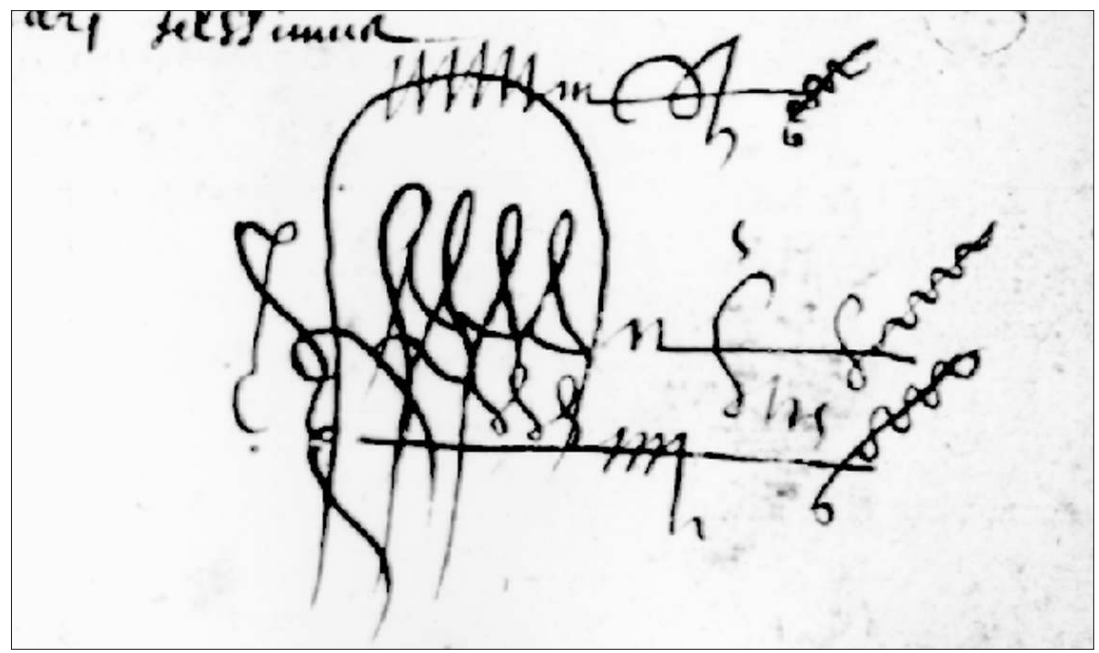


Saint-Martin de Tours et les chancelleries carolingiennes

Figure 5 : Florence, Bibl. Laurenziana, Ms. Ashburnham 1836, $f^{\circ} 6 v^{\circ}$ (cliché : MGH Apparat Ludwig der Fromme, Bonn)

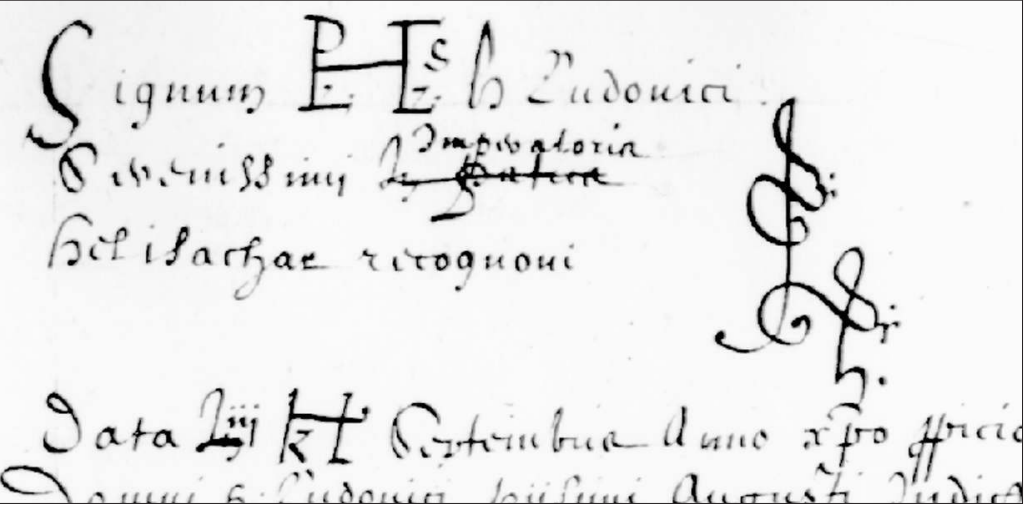

Figure 6 - Florence, Bibl. Laurenziana, Ms. Ashburnham 1836, f.38 $r^{\circ}$ (cliché : MGH Apparat Ludwig der Fromme, Bonn)

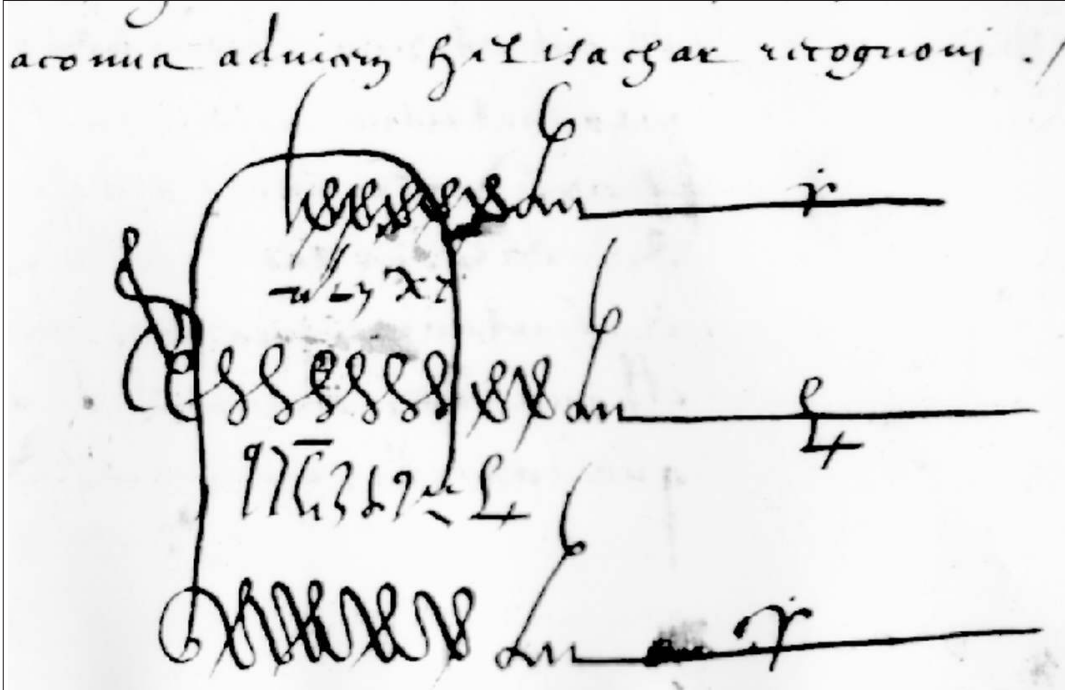

87 
Figure 7 : Florence, Bibl. Laurenziana, Ms. Ashburnham 1836, $f^{\circ} 39 v^{\circ}$ (cliché : MGH Apparat Ludwig der Fromme, Bonn)

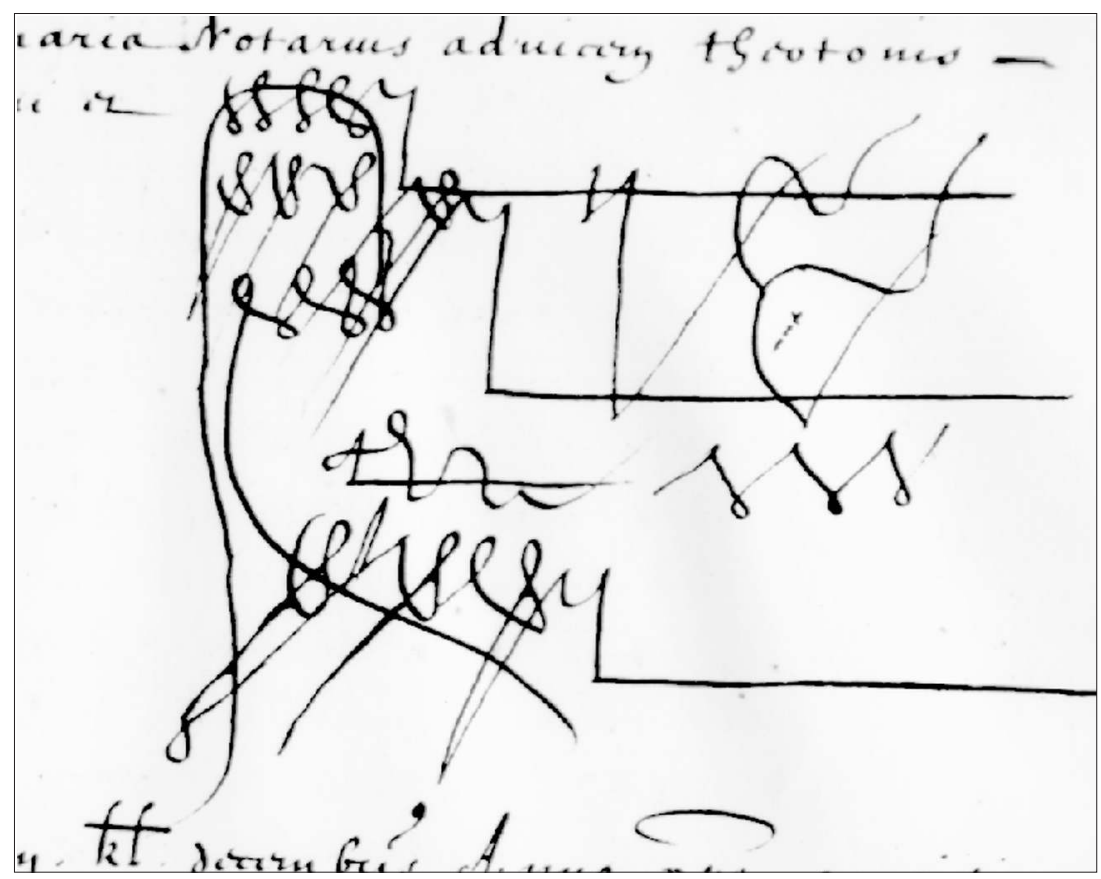


Figure 8 - Florence, Bibl. Laurenziana, Ms. Ashburnham 1836, $f^{\circ} 38 v^{\circ}$ (cliché : MGH Apparat Ludwig der Fromme, Bonn)

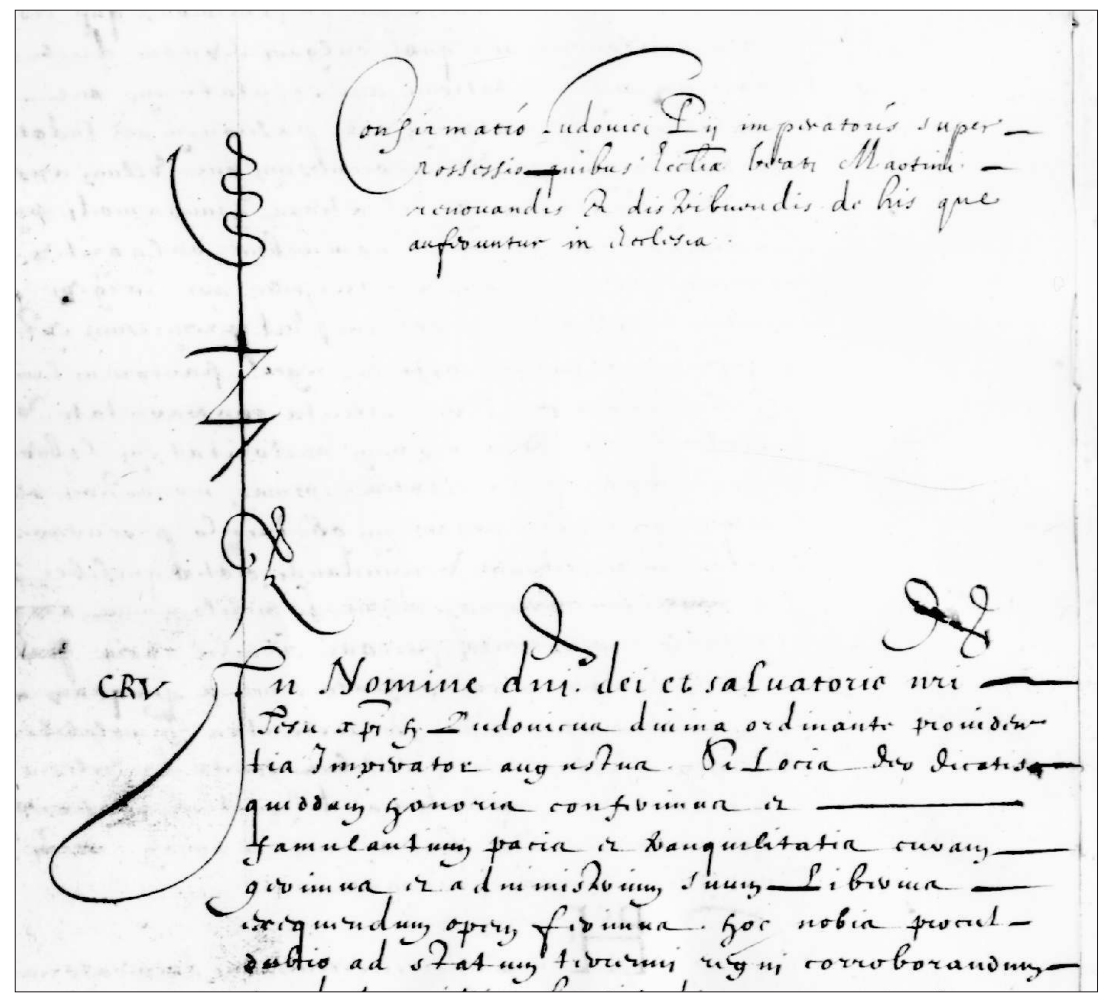

Figure 9 - Florence, Bibl. Laurenziana, Ms. Ashburnham 1836, $f^{\circ} 34 r^{\circ}$ (cliché : MGH Apparat Ludwig der Fromme, Bonn)

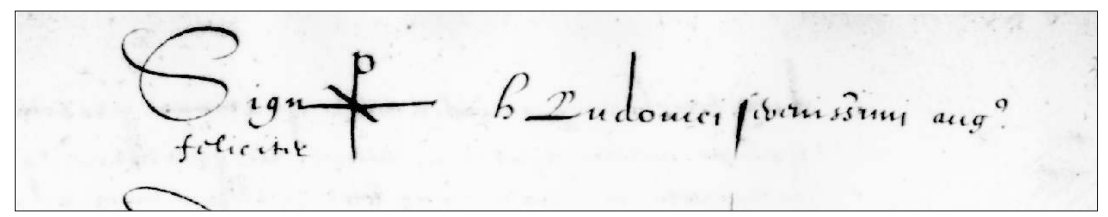




\section{RESUME}

L'état de la documentation interdit une analyse approfondie sur SaintMartin de Tours et les chancelleries carolingiennes; on ne peut s'appuyer que sur les copies d'érudits faites au $\mathrm{XVII}^{\mathrm{e}}$ et au XVIII ${ }^{\mathrm{e}}$ siècle. Certains dessins permettent toutefois d'étudier la pratique médiévale de cotation à Tours; il est également possible d'identifier les signes graphiques typiques des notaires qui ont reconnu les diplômes. Sous les règnes de Charlemagne et de Louis le Pieux, la rédaction des actes par le destinataire était exceptionnelle. Ce fut cependant le cas pour une partie des diplômes de Louis le Pieux pour SaintMartin. Des relations personnelles très étroites existaient alors entre cet établissement et la chancellerie impériale. Le manuscrit latin 2718 de la Bibliothèque nationale de France en constitue le monument le plus important; il s'agit d'une collection occasionnelle, et non systématique, rédigée à la chancellerie en utilisant des chutes de parchemin. On ignore l'identité des scribes.

\section{ABSTRACT}

The transmission allows no profound examination of Saint-Martin and the Carolingian chanceries, because all have to base upon the copies of antiquarian researchers of the $17^{\text {th }}$ and $18^{\text {th }}$ centuries. Some drawings allow to examine the medieval practise of archival notation at Tours, it is also possible to identify the typical graphical signes of the notaries who have recognized the charters. During the reign of Charlemagne and Louis the Pious the composing and drafting of diplomas by the beneficiaries was extraordinary, but indeed it is the case for some charters of Louis the Pious for Saint-Martin. There were strong relations of personnel between the imperial chanceries and the chapter. The most important monument of these relations is the famous manuscript Latin 2718 of the Bibliothèque nationale de France, a occasional, not systematic collection drafted in the chancery using left over parchment. The scribes could not be identified yet. 